\title{
A modular high-throughput in vivo screening platform based on chimeric bacterial receptors
}

\author{
Christina E. Lehning ${ }^{1}$, Jan B. Heidelberger ${ }^{2}$, John Reinhard ${ }^{3}$, Morten H. H. Nørholm ${ }^{1}$
} and Roger R. Draheim ${ }^{4,5 *}$

${ }^{1}$ Novo Nordisk Foundation Center for Biosustainability, Technical University of Denmark, Building 220, Kemitorvet, DK-2800, Kgs. Lyngby, Denmark, ${ }^{2}$ Institute of Molecular Biology, Ackermannweg 4, D-55128, Mainz, Germany, ${ }^{3}$ Buchmann Institute for Molecular Life Sciences, Goethe University Frankfurt, Max-von-Laue-Straße 15, D-60438, Frankfurt, Germany, ${ }^{4}$ School of Pharmacy and Biomedical Sciences and ${ }^{5}$ Institute of Biomedical and Biomolecular Science, University of Portsmouth, White Swan Road, PO1 2DT, Portsmouth, United Kingdom

Running title: High-throughput screening with tuned chimeric bacterial receptors

*To whom correspondence should be addressed (R. R. D.):

University of Portsmouth

School of Pharmacy and Biomedical Sciences

St. Michael's Building

White Swan Road

Portsmouth

PO1 2DT

United Kingdom

tel: $+44(0) 2392842133$

fax: +44 (0)2392843565 


\section{Abstract}

Multidrug resistance (MDR) is a globally relevant problem that requires novel approaches. Two-component systems are a promising, yet untapped target for novel antibacterials. They are prevalent in bacteria and absent in mammals, and their activity can be modulated upon perception of various stimuli. Screening pre-existing compound libraries could reveal small molecules that inhibit stimulus-perception by virulence-modulating receptors, reduce signal output from essential receptors or identify artificial stimulatory ligands for novel SHKs that are involved in virulence. Those small molecules could possess desirable therapeutic properties to combat MDR. We propose that a modular screening platform in which the periplasmic domain of the targeted receptors are fused to the cytoplasmic domain of a well-characterized receptor that governs fluorescence reporter genes could be employed to rapidly screen currently existing small molecule libraries. Here, we have examined two previously created Tar-EnvZ chimeras and a novel NarX-EnvZ chimera. We demonstrate that it is possible to couple periplasmic stimulus-perceiving domains to an invariable cytoplasmic domain that governs transcription of a dynamic fluorescent reporter system. Furthermore, we show that aromatic tuning, or repositioning the aromatic residues at the end of the second transmembrane helix (TM2), modulates baseline signal output from the tested chimeras and even restores output from a non-functional NarX-EnvZ chimera. Finally, we observe an inverse correlation between baseline signal output and the degree of response to cognate stimuli. In summary, we propose that the platform described here, a fluorescent Escherichia coli reporter strain with plasmid-based expression of the aromatically tuned chimeric receptors, represents a synthetic biology approach to rapidly screen preexisting compound libraries for receptor-modulating activities.

\section{Keywords}


Receptor engineering / chimeric receptors / aromatic tuning / high-throughput screening / biological platforms 
Multidrug resistance (MDR) is a problem that is frequently associated with nosocomial infections and that limits therapeutic options. ${ }^{1}$ In Europe, infections with antibiotic-resistant bacteria kill nearly 25,000 patients annually and represents a total expenditure of approximately 1.5 billion $€^{2}$ The discovery and adaptation of traditional antibiotics has stagnated in recent decades. It is thus important to search for novel approaches to combat the growing danger of antibiotic resistance.

Bacteria sense, respond and adapt to external stimuli via two-component signaling (TCS) circuits. The external stimuli comprise a wide range of environmental conditions including nutrient availability, ambient temperature or external osmolarity. ${ }^{3}$ Another purpose of TCSs is to facilitate multi-organism phenomena such as quorum sensing, biofilm formation and host-pathogen interaction. ${ }^{4}$ Most importantly, TCSs are widespread in bacteria but noticeably absent in mammals, which renders them excellent targets for novel antibacterials. ${ }^{5}$

A canonical TCS consists of a membrane-spanning sensor histidine kinase (SHK) and a cytoplasmic response regulator (RR). ${ }^{3}$ The largest group of SHKs possesses a periplasmic or extracellular domain responsible for stimulus perception. Interaction with stimuli provokes subsequent signal transduction to the cell interior via conformational charges of the adjacent transmembrane domain. ${ }^{6}$ These conformational changes trigger autophosphorylation and subsequent phosphorylation of the RR. Within bifunctional SHKs, the extent of input stimulus controls the ratio of the kinase and phosphatase activities and thus governs the intracellular level of phosphorylated cognate RR. ${ }^{7}$ This phosphorylation, in turn, modulates the activity of the attached output domain, which interacts with DNA to control transcription of genes appropriate for mediating a response to the perceived stimulus. ${ }^{3}$

Based on the conserved domain function of TCSs, two different classes of therapeutic targets have been proposed, each with its own supporting evidence. ${ }^{8}$ Several SHKs that regulate bacterial virulence and their respective cognate stimuli are known and a previous study suggests that SHKstimulus interactions can be disrupted. ${ }^{9}$ QseC is a canonical SHK found within at least 25 important 
human and plant pathogens. Screening a library of 150,000 small organic molecules resulted in several hits that inhibited QseC-mediated expression of downstream virulence genes. The most promising of these compounds (LED209) was shown to reduce the efficiency of colonization by several QseC-containing pathogens in mice. ${ }^{9}$ A subsequent study employed structure-activity relationships (SAR) to identify several more effective derivatives of LED209. ${ }^{10}$ A similar approach led to a discovery of an inhibitor targeting FsrC, an SHK from the Gram-positive Enterobacter faecalis that is found in the human gut. Vancomycin-resistant E. faecalis (VRE) has recently become a severe clinical problem. ${ }^{11}$ Gelatinase biosynthesis-activating pheromone (GBAP), a small cyclic lactone-containing peptide, has been shown to be the cognate ligand of FsrC. ${ }^{12}$ In order to find an appropriate inhibitor, actinomycete culture supernatants were screened, resulting in the discovery of Siamycin I, which suppressed GBAP-based signal transduction by FsrC. ${ }^{13-14}$ In addition, the AgrCAIP (autoinducing peptide) pairs from Staphylococcus aureus can be divided into four agr specificity groups. On the same SHK, non-cognate AIPs work antagonistically against the cognate ligands. These results demonstrate the feasibility of finding small molecules that inhibit responses to physiological stimuli. ${ }^{15}$ These virulence-attenuating compounds, which we term Class I compounds, can be discovered within pre-existing libraries of small molecules.

The second mechanism of TCS targeting involves direct inhibition of signal output from essential SHKs. An example target would be the WalK SHK, which has been shown to be essential in several Gram-positive species. ${ }^{16}$ Several inhibitors of WalK have been previously identified by complementary screening methods involving synthetic libraries and cell lysates. ${ }^{17-19}$ In these cases, an example of what we call Class II compounds have shown to have specific interaction with WalK that lead to cell death in Bacillus subtilis and Staphylococcus aureus. In a similar manner, HP0165 of Helicobacter pylori and MtrB of Mycobacterium tuberculosis are also essential for cell viability and could be targeted in a similar manner. ${ }^{20-21}$ 
We propose a modular screening platform that can rapidly test libraries of small molecules to facilitate identification of inhibitors of stimulus perception (Class I) or compounds that directly inhibit signal output from essential SHKs (Class II). In addition, we propose that this platform can be used to identify natural or artificial stimulatory ligands for SHKs that are involved in virulence. The central idea is that the periplasmic domain of the SHK of interest is fused to the platform that comprises the cytoplasmic domain of a well-characterized SHK, which in turn governs fluorescent reporter genes for a fast, reliable and detectable signal. In this study, we have physically coupled the periplasmic stimulus-perceiving domains from bacterial receptors (chemoreceptors or SHKs) to the cytoplasmic domain from the major osmosensor of E. coli (EnvZ), which is part of a wellcharacterized signaling pathway. This approach allowed us to directly couple stimulus-perception by the periplasmic domain of the aspartate chemoreceptor (Tar) from E. coli to an EnvZ-based signaling pathway that controls transcription of cyan fluorescent protein (CFP) and yellow fluorescent protein (YFP). This synthetic circuit facilitated detection of changes in chimeric signal output by monitoring whole-cell CFP and YFP fluorescence by spectrofluorometery or on a single-cell level by flow cytometry. We also employed aromatic tuning ${ }^{22-23}$ to modulate the signal output of two different TarEnvZ (Taz1 and Tez1A1) chimeras. In some cases, this tuning resulted in a change in baseline signal output while retaining the sensitivity to aspartate. The value of aromatic tuning became evident when we created a novel chimera composed of the periplasmic domain of the nitrate/nitrite sensor of E. coli (NarX) and the cytoplasmic domain of EnvZ, which possessed no signal output until aromatic tuning was employed. We would like to emphasize that the screening platform is optimized for automated flow cytometry, which can facilitate rapid screening of pre-existing compound libraries for novel signal-modulating compounds that possess antibacterial properties.

\section{Results and Discussion}

Re-creation of two Tar-EnvZ chimeras (Tazl and Tez1A1) 
TCSs must detect a wide variety of stimuli in order to control of a diverse array of bacterial processes. Within an evolutionary context, this has been accomplished by altering the stimulussensing properties of the periplasmic domains of SHKs and the DNA-binding specificity of RR output domains, while leaving the remainder of the TCS scaffold fundamentally unchanged (Figure 1A). Within canonical homodimeric SHKs, a common membrane-spanning topology is observed that consists of a cytoplasmic N-terminus, the first transmembrane helix (TM1), a periplasmic stimulusperceiving domain, the second transmembrane helix (TM2), the cytoplasmic dimerization and histidyl phosphotransfer domain (DHp), and the C-terminal catalytic ATPase (CA) domain (Figure 1B). The modular screening platform presented here exploits the conservation observed within the TM and HAMP (originally identified within histidine kinases, adenylate cyclases, MAP kinases and phosphatases) domains to physically couple periplasmic stimulus-perceiving domains from targeted SHKs to cytoplasmic domains with well-characterized signaling pathways to produce chimeric SHKs. This allowed us to directly couple stimulus-perception to stimulation of a well-characterized EnvZ-based signaling pathway. ${ }^{24-27}$

We began by generating previously described functional chimeric receptors containing the periplasmic and transmembrane domains of the aspartate chemoreceptor (Tar) and the cytoplasmic signaling domains of the major osmosensor of E. coli (EnvZ, Figures $2 \mathrm{~A}$ and $2 \mathrm{~B}) .{ }^{28-29}$ An additional advantage to this system is that the cytoplasmic domain of EnvZ $\left(E_{n} Z_{c}\right)$ has been previously shown to be sufficient for osmosensing. ${ }^{30-32}$ Therefore, we can easily determine whether the osmosensing functionality can be retained in chimeras possessing a periplasmic domain that sense a stimulus not directly related to osmosensing.

The fusion point of the chimeric Taz1 receptor is located near the C-terminus of the HAMP domain, resulting in both transmembrane helices (TM1 and TM2) and the majority of the HAMP domain being contributed from Tar, while the remainder of the chimera is from EnvZ. ${ }^{28}$ Tez1A1 is 
similar to Taz1, except that the fusion point is not within the HAMP domain but rather at the cytoplasmic end of TM2. ${ }^{29}$ To restore functional coupling between Tar and EnvZ within Tez1A1, an additional alanine residue (Ala-213) was introduced immediately C-terminal to the final residue contributed by Tar (Ile-212). ${ }^{29}$ Consequently, the two transmembrane helices of the receptor are supplied by Tar, while the entire cytoplasmic domain originates from EnvZ (Figures $2 \mathrm{~A}$ and 2B). It should also be noted that we added a short C-terminal heptaresidue linker and V5-epitope tag in order to assess expression levels. We have previously shown that this tag does not alter the signal output from wild-type EnvZ. ${ }^{22}$

Modulation of signal output from Tazl and Tez1A1 in response to osmolarity and aspartate

MDG147 is a dual-color fluorescent reporter strain that has been previously used to quantify transcription regulated by the EnvZ/OmpR TCS. The strain is a derivative of K-12 MG1655 that possesses transcriptional fusions of $c f p$ to $\operatorname{ompC}$ and of $y f p$ to $o m p F .^{33}$ Therefore, the level of intracellular phosphorylated OmpR can be estimated by quantifying the ratio of CFP to YFP fluorescence (Figure S1A). As previously described, MDG147/pEB5 cells demonstrated elevated levels of EnvZ signal output as measured by CFP/YFP ratio when cells were grown under the high osmolarioty regime (15\% sucrose) compared to when grown under the low osmolarity regime $(0 \%$ sucrose) (Figures S1B and S1C). ${ }^{22,33-34}$ Strain EPB30 $0^{35}$ is a $\triangle e n v Z$ derivative of MDG147 that is suitable for assessing the signal output upon plasmid-based expression of the tar-envZ genes. A band was observed that represented the Tar-EnvZ chimera from EPB30/pRD500 and EPB30/pRD501 cells expressing Taz1 or Tez1A1 respectively, whereas control strains not expressing the V5-tagged EnvZ did not produce this band (Figure S2). These bands demonstrate that the chimeras are stably expressed and suitable for subsequent analysis. 
We continued by measuring the relative signal output from EnvZ-deficient circuits containing either Taz1 or Tez1A1. This was accomplished by comparing CFP and YFP fluorescence from cells expressing Taz1 (EPB30/pRD500) or Tez1A1 (EPB30/pRD501) with fluorescence from cells expressing wild-type EnvZ (MDG147/pEB5) or $\Delta e n v Z$ cells (EPB30/pEB5). We determined that circuits containing either Tar-EnvZ chimera exhibited CFP and YFP levels greater than $\Delta e n v Z$ cells. This demonstrates that the chimeras were able to phosphorylate OmpR in the absence of aspartate, the cognate ligand for the Tar periplasmic domain. Furthermore, a change in CFP/YFP ratio was observed when these cells were grown under the high-osmolarity regime, albeit to a smaller degree than wild-type cells (Figure S3). Thus, external osmolarity appears to modulate signal output from both Tar-EnvZ chimeras, which confirms previous results suggesting that external osmolarity is sensed and processed by the cytoplasmic domain of EnvZ. ${ }^{30-31}$

To examine response to aspartate, EPB30/pRD500 or EPB30/pRD501 cells expressing Taz1 or Tez1A1, respectively, were subjected to increasing concentrations of aspartate. For both receptors, we observed an increase in CFP fluorescence (Figure S4A), a decrease in YFP fluorescence (Figure S4B) and an overall increase in the CFP/YFP ratio (Figure 3A). Leucine or isoleucine, present at 10 $\mathrm{mM}$ as controls, showed minimal effect on signal output from these circuits (Figure 3A). It should be noted that this result is in disagreement with previously published data demonstrating that leucine decreases Taz1 signal output. ${ }^{36}$ In addition, the CFP/YFP ratio curves are parallel for both Tar-EnvZ chimeras, indicating that they responded to aspartate in a similar manner, although Tez1A1 possessed a slightly elevated signal output across the entire curve. When the increase from baseline was calculated, circuits containing Taz1 possessed a greater dynamic range (about a 30-fold increase in CFP/YFP ratio) compared to those containing Tez1A1 (about a five-fold increase in CFP/YFP ratio). These results clearly demonstrate the value in producing chimeras with minimal baseline signal output in applications requiring detection of an increase in CFP/YFP ratio due to stimulus-perception (Figure 3B). In circuits containing Tez1A1, it appears that CFP fluorescence becomes saturated, an 
effect that limits the overall dynamic range of circuits containing this chimera (Figure S4). In summary, these results indicate that both Taz1 and Tez1A1 respond to aspartate and demonstrate that our synthetic circuits with Tar-EnvZ chimeras function as expected based on previous results.

\section{Aromatic tuning of Tazl and Tez1A1}

As shown in Figure 3, not only is it necessary to design chimeras in which the stimulusperceiving domain is functionally coupled to the intracellular signaling domains; it is also important to ensure that the baseline signal output is appropriate for the desired application, which means that the fluorescent output of the reporter proteins does not saturate. For example, when screening a compound library for small molecules that inhibit stimulus-perception (Class I), it is advantageous to use chimeras that possess a low baseline signal output that significantly increases upon addition of the cognate stimulus. In this regard, Figure 3 clearly demonstrates that using Taz1 is superior to Tez1A1 within a synthetic circuit. This parameter comes into play within a screening platform that aims to detect Class I compounds that prevent perception of aspartate by the periplasmic domain of the Tar-EnvZ chimeras. Conversely, when screening for Class II small molecules that inhibit signal output from an essential SHK, a higher baseline signal output would be favorable as a decrease of signal output upon binding of the inhibitor must be detectable. In addition, when screening for natural or artificial ligands of SHKs involved in virulence, it would be critical to have a low baseline signal output.

Based on the "regulated unfolding" model of signal transduction ${ }^{37}$, which proposes that signaling within a receptor is guided by unfolding of individual receptor subdomains, the TM2HAMP junction (Figure 1B) is an ideal position to target with the aim of mimicking signal transduction due to stimulus-perception. We have shown that moving aromatic residues about their original position, by site-directed mutagenesis, at the C-terminal end of TM2 and adjacent to the 
TM2-HAMP interface, modulates signal output from the aspartate chemoreceptor of E. coli (Tar) and from full-length EnvZ $Z^{22-23,38}$. In order to manipulate baseline signal output, we examined whether aromatic tuning could be employed within the context of the Tar-EnvZ chimeras. Taz1 and Tez1A1 were modified by repositioning a Trp-Tyr tandem pair about its original position at the cytoplasmic end of the TM2 domain of Tar (Figure 4A). ${ }^{38}$ We began by expressing the tuned chimeras from pRD500 (Taz1) and pRD501 (Tez1A1), respectively, in EPB30 cells and monitored their expression. When EPB30 cells were grown under the low-osmolarity regime, we found that the WY-3 and WY+1 variants of Taz1 and the WY+1 variant of Tez1A1 were unstable (Figures S5A and S5B). When the EPB30 cells were grown under the high-osmolarity regime, only the WY+1 variants of Taz1 and Tez1A1 were unstable. Thus, the WY-3 variant of Taz1 was differentially stable based upon the growth regime that was employed. In addition, the degradation appeared to be a two-step process (Figure S5C). Receptors that exhibited degradation, e.g. Taz1 WY-3, Taz1 WY+1 and Tez1A1 $\mathrm{WY}+1$, were not analyzed further.

We observed that aromatic tuning modified signal output from the chimeras, as evidenced by a change in steady-state signal output (Figure 4B, CFP/YFP ratio). As seen with full-length EnvZ, ${ }^{22}$ the pattern of signal outputs from EPB30/pRD500 cells expressing the tuned variants of Taz1 follows a helical pattern, suggesting that the TM2 surface that the aromatic residues reside upon is of critical importance (Figures 4B and S6). A possible explanation for this observation is that the rotational movement of the aromatic residues around the TM2 surface results in disruptions to the helical packing of the transmembrane domain as proposed previously within EnvZ. ${ }^{22}$ For example, the WY2 and $\mathrm{WY}+2$ variants represent local signal output maxima, whereas WY-1 and WY+3 possess lower signal output, and the wild-type results in the lowest fluorescent output. Also consistent with these results are the observations the WY-3 and WY+1 variants were subject to degradation (Figure S5), an effect that may reflect disorder in the packing of TM2.

The results with cells expressing the tuned variants of Tez1A1 were similar, but the entire set 
possessed greater levels of signal output compared to their Taz1 counterparts (Figure 4B). In most cases, the dynamic range of the aromatically tuned chimeras in response to external osmolarity were similar, with a four-fold to five-fold increase in CFP/YFP ratio for the Taz1 family and between a two-fold and four-fold increase for the Tez1A1 family (Figure 4C). As shown in Figure S6, the changes in CFP/YFP ratio were due to both an increase in CFP fluorescence and a concomitant decrease in YFP fluorescence. The notable exception was the wild-type Taz1, which showed a smaller dynamic range than the other tuned Taz1-containing circuits, likely because the extremely low CFP fluorescence values obscure any real differences in the signal (Figure S6).

\section{Response of the aromatically tuned Tazl and TezlA1 chimeras to aspartate}

We expressed the aromatically tuned Taz1 and Tez1A1 variants in EPB30/pRD500 or EPB30/pRD501 cells, respectively, and monitored changes in signal output in response to increasing concentrations of aspartate. In all cases, the surface that the aromatic residues reside upon governed signal output. For the Taz1 family of tuned chimeras, the wild-type Taz1 and the WY+3 variants exhibited the greatest increases in signal output, as measured in fold-change in CFP/YFP ratio. The next most responsive to aspartate were the WY-1 and WY+2 variants, which also reside on the same helical surface, whereas WY-2 did not demonstrate an increase. It is also important to note that two chimeras that were subject to degradation (Taz1 WY-3 and WY+1) also reside on the same surface of TM2 (Figures 5 and S7). Assessing the family as a whole, an inverse correlation was observed between steady-state signal output and the magnitude of response to aspartate. In other words, the lower the steady-state CFP/YFP signal output, the greater the increase in signal output in response to increasing aspartate concentrations (Figures 5 and S7).

The aromatically tuned variants of Tez1A1 behaved differently than the Taz1-based family in response to saturating aspartate. The minus variants (WY-3 through WY-1) were all stable and 
strongly activated, as shown by elevated CFP levels (Figure S7), reduced YFP levels (Figure S7) and high CFP/YFP ratios (Figure 5). The WY+1 variant was subject to cleavage, whereas the unmodified Tez1A1 and the WY+2 and WY+3 variants responded to aspartate (Figures 5 and S7).

In order to test the applicability of this platform for high-throughput screening, we compared the results of this experiment with experiments performed with flow cytometery. Besides a drastically improved throughput, flow cytometry also provides an additional level of resolution, allowing the fluorescent signal from single cells rather than entire populations to be observed. For the tuned Taz1 and Tez1A1 families, we observed similar relative baseline signal output (CFP/YFP ratio) and response to saturating aspartate concentrations (medium gray). Isoleucine at $10 \mathrm{mM}$ (white) was employed as a control and showed comparable ratios to baseline activity (dark gray; Figure 6). Both methodologies show a significant $(p<0.001)$ increase in signal output from several receptors upon addition of aspartate. This result demonstrates that analysis via automated flow cytometry is a viable method for high-throughput screening (Figure 6).

\section{Creation and analysis of tuned NarX-EnvZ (NavZ1) chimeras}

In order to demonstrate that the aforementioned methods were not restricted to the Tar-EnvZ chimeras, we created a novel protein, which we termed NavZ1, that contains the periplasmic sensor, transmembrane domains and of HAMP domain of the nitrate/nitrite sensor of E. coli (NarX) fused to the cytoplasmic signaling domain of the sensor histidine kinase EnvZ. The fusion joint was within the HAMP domain and in the same relative position as the fusion joint in Taz1 (Figures 7A and 7B). A catatg (His-Met) NdeI restriction site was introduced at the C-terminal end of the NarX fragment to create the fusion point at the same location as in the Tar-EnvZ chimeras. Expression of Naz1 from pRD502 in EPB30 cells demonstrated the presence of intact NavZ1 (Figure S8). However, the unstimulated fluorescence signal of CFP and YFP was lower than that of EPB30/pEB5 ( $\triangle e n v Z$ ) cells, 
showing that signal output was insufficient to generate enough phospho-OmpR to result in $o m p F$ and $y f p$ transcription (Figure 7C). Thus, we did not further analyze the un-tuned NavZ1.

To assess whether aromatic tuning can restore activity, we moved the NarX-derived Trp-173 residue of NavZ1 about its original position near the cytoplasmic end of TM2, resulting in the W-3 through W+3 variants (Figure 7D). Unlike Taz1 and Tez1A1, all aromatically tuned variants were expressed stably (Figure S8), and all of them, when expressed in EPB30/pRD502 (NavZ1) cells, supported higher levels of CFP or YFP fluorescence than EPB30/pEB5 (AenvZ). Thus, aromatic tuning can restore the activity of a non-functional chimera.

The minus-series of NavZ1 variants, W-3 through W-1, produced YFP fluorescence similar to cells expressing Taz1, while the plus-series of mutants, $\mathrm{W}+1$ through $\mathrm{W}+3$, exhibited even higher signal output than the minus-series. In addition, osmolarity modulated signal output from all NavZ1 chimeras that were analyzed (Figures 7E and S9). It should also be noted that local minima/maxima were observed as the Trp residue was repositioned around the surface of TM2.

Several lines of research have demonstrated that when aspartate binds to Tar, a displacement of TM2 on the order of 1-3 $\AA$ toward the cytoplasm occurs. ${ }^{38-45}$ The final result is a decrease in CheA kinase activity that leads to counterclockwise $(\mathrm{CCW})$ flagellar rotation, which constitutes an attractant response. ${ }^{46-49}$ In contrast, previous analyses of a NarX-Tar chimera, demonstrated that nitrate elicits clockwise $(\mathrm{CW})$ flagellar rotation, which is indicative of a repellent response. ${ }^{50-51}$ Consistent with this observation, crystallographic structures of the periplasmic domain of NarX demonstrate a relative displacement between the $\mathrm{N}$ - and $\mathrm{C}$-terminal helices (helices 1 and 4) of the NarX sesnsor domain that is opposite of the one observed in the crystallographic structure of the periplasmic of Tar. ${ }^{43,52}$ Based on these results, we predicted a reduction in NavZ1 signal output upon addition of nitrate, rather than the increases observed with Taz1 and Tez1A1 after addition of aspartate. 
As described above, it is important for tuned chimeric receptors to possess signal output appropriate for the assay to be performed. Therefore, we predicted that observation of a decrease in signal output of NavZ1 would require a high-steady state fluorescence signal. The W-3, W-2, W-1, wild-type and W+2 NavZ1 variants did not fulfill this requirement.

For this application, we employed automated flow cytometry because adding $10 \mathrm{mM} \mathrm{KNO}_{3}$ dramatically increased the growth rate of EPB30/pRD502 (NavZ1) cells relative to cultures containing $10 \mathrm{mM} \mathrm{KCl}$, thus casting doubt on the reliability of experiments using spectrophotometry with bulk cultures. This problem is less of a concern during flow cytometric analysis. The steadystate signal output from unstimulated cells analyzed by spectrophotometry (MMA; Figure 8A) was found to be similar across the family of tuned variants when compared to those analyzed by flow cytometry (MMA; Figure 8B). These results gave us confidence in the usage of flow cytometry for analysis of signal output by the NavZ1 chimeras. When $10 \mathrm{mM} \mathrm{KNO}_{3}$ was used as the cognate ligand, we observed a significant $(p<0.001)$ decrease in signal output from the tuned $\mathrm{W}+1$ variant (Figure $8 B$ ), while the $W+3$ variant did not exhibit such a decrease, giving us confidence that the decrease in signal output with the $\mathrm{W}+1$ receptor was due to decreased signaling rather than a pleiotropic effect on cell metabolism. These results demonstrate that a stable NarX-EnvZ chimera can be created and that aromatic tuning can restore fluorescence signal output and generate receptors $(\mathrm{W}+1$ in this case) that are useful for monitoring changes in response to stimuli.

\section{Summary and concluding remarks}

In summary, the majority of the aromatically tuned Tar-EnvZ receptors were stably expressed and responded to aspartate to an extent that correlates inversely with their baseline signal output. We found that the fusion site of Taz1, which is within the C-terminus of the HAMP domain, is more amenable for the rational chimera design than that site employed within Tez1A1, which is 
immediately C-terminal to TM2. This result suggests that it may be important to have both TM2 and the HAMP domain contributed by the receptor to be tested. This is finding is reasonable, as direct communication between TM2 and HAMP has been shown to be important for the signaling properties of chemoreceptors. ${ }^{53-55} \mathrm{We}$ also showed that we could, through aromatic tuning, generate a NarXEnvZ chimera that responded to the addition of nitrate, the most effective ligand for NarX. Finally, we showed that flow cytometry should be applicable to the screening of large libraries of possible ligands, whether agonistic or antagonistic, for any given receptor.

Two previously studied Tar-EnvZ chimeras (Taz1 and Tez1A1) were functional within our experimental platform as measured by population-level microplate fluorometry and flow cytometry on a single-cell level. Both chimeras were expressed, embedded within the membrane and mediate response to aspartate. Aromatic tuning was employed within the chimeras with varying success, as different tuned chimeras had various initial steady-state signal outputs. An inverse correlation was observed between the extent of baseline signal output and the degree of response to aspartate. Finally, we created a novel NarX-EnvZ (NavZ1) chimera that could be aromatically tuned to restore signal output to a non-functional chimera while responding to stimulus. A recent paper demonstrated production of function and non-functional chimeric chemoreceptors with different fusion points. ${ }^{56}$ We believe that employment of aromatic tuning as described here would restore signal output to the non-functional chimeras.

We propose that strain EPB30 and plasmid-based chimeric receptor expression from a suitable derivative of pRD500 can be employed as a modular high-throughput screening platform. In the case of Class I SHK targets, we have shown with the tuned Taz1 and Tez1A1 chimeras that a minimal baseline signal output is desirable, such that the cognate ligand facilitates the greatest dynamic range possible. This situation maximizes the ability of these synthetic circuits to respond to inhibition of their activity by a relevant stimulus and allows us to identity such perception-inhibiting compounds. A recent review nicely summarizes virulence-attenuation involving what we term Class I SHK 
targets. ${ }^{57}$ With Class II SHK targets, it was shown to be critical to have the maximal possible signal output so that even a small decrease could be observed during screening. We propose that such a platform could be employed to identify novel compounds that interact with the targeted periplasmic domain. It is important to note that aromatic tuning resurrected the "dead" NavZ1 chimera to allow nitrate to be identified as significantly decreasing $(25 \%, p<0.001)$ signal output.

In conclusion, the work described here introduces a modular platform that is amenable to interchangable periplasmic domains and screening libraries of small molecules. We propose that this platform has several advantages over previous screening methods. Firstly, it will facilitate direct identification of compound:SHK pairs due to a single chimera governing transcription of ompC and $o m p F$, and thus CFP and YFP fluorescence, of which changes can be easily identified. This is major advantage compared to standard in vivo screening that requires expensive and time-consuming subsequent target validation. Second, in the long-term, this platform will allow targets, namely SHKs from multidrug resistant (MDR) organisms, to be screened in non-pathogenic laboratory strains such as E. coli EPB30. This ability will greatly expand the spectrum of research groups that can participate in antibacterial screening by reducing the initial requirement for ACDP Category 3 or 4 facilities. In addition, it makes targeting SHKs from an organism solely based on its annotated genome sequence possible. Third, the platform only screens molecules that interact only with the periplasmic domain of the SHK that is fused to EnvZ, which eliminates the necessity for exogenous compounds to cross the cytoplasmic membrane. Finally, the ability to employ one method on a wide range of targets would standardize screening results, with the resulting benefit of being able to compare results for an entire family of related SHKs or with libraries of related compounds from a library of small molecules.

\section{Methods}


For all DNA manipulations, E. coli strain MC1061 ${ }^{58}$ was used. To control for light scattering and cellular autofluorescence during population-based measurements, strain MG1655 was employed. Strains MDG147 [MG1655 $\left.\Phi\left(o m p F^{+}-y f p^{+}\right) \Phi\left(o m p C^{+}-c f p^{+}\right)\right]^{33}$ and EPB30 (MDG147 envZ::kan $)^{35}$ were used for analysis of steady-state signal output in measurements at both the population and single-cell levels. Plasmids used for chimeric receptor expression were derived from $\mathrm{pRD} 400^{22}$ in order to retain the same IPTG-based induction of receptor expression. Initially, the N-terminal section of Tar was amplified from pMK113V5 $5^{39}$ in a manner that facilitated subcloning of residues Met-1 through His-243, which resulted in pRD500 that expresses Taz1-V5 upon induction with IPTG. It was previously shown that the V5-eiptope tag has no adverse effect on EnvZ or Tar signal output. ${ }^{22}$, 34, 39, 55, 59-61 Standard site-directed mutagenesis techniques were employed to move the Trp-Tyr pair about their original position. A USER-based cloning method ${ }^{62-63}$ was used to create pRD501 from which Tez1A1 was expressed. The advantage of the method is that the aromatic tuning and the addition of the Ala after the end of TM2 were created simultaneously. Finally, standard molecular biological methods were employed to amplify the $\operatorname{narX}$ gene from strain BW25113 and subclone residues Met-1 through Asn-217 into pRD500. This required a N218H substitution within the NarX component in order to create an NdeI resturction site for molecular cloning. The final product, pRD502, expresses NavZ1 upon induction with IPTG in a similar manner to the above chimeras. Site-directed mutagenesis was carried out as above to aromatically tune (Trp-173) the NarX-EnvZ chimera. A previously described plasmid, $\mathrm{pEB5}^{64}$ served as an empty vector control that did not express envZ. 
Analysis was performed as described previously $y^{22,64}$ with slight modification. Fresh colonies of MDG147 ${ }^{33}$ or EPB30 35 transformed with pEB5 $^{64}$, pRD500 (Taz1), pRD501 (Tez1A1) or pRD502 (NavZ1) as necessary were used to start 2-ml overnight culture of minimal medium $\mathrm{A}^{65}$ supplemented with $0.2 \%$ glucose, $50 \mu \mathrm{g} / \mathrm{ml}$ ampicillin and $20 \mu \mathrm{m}$ IPTG. When cells were grown under the high osmolarity regime, $15 \%$ sucrose was present. Aspartate, leucine or isoleucine was present as indicated. After growing overnight at $37^{\circ} \mathrm{C}$, cells were diluted at least 1:1000 into $7 \mathrm{ml}$ of fresh medium. When cultures reached an $\mathrm{OD}_{600 \mathrm{~nm}} \sim 0.3$, chloramphenicol was added to a final concentration of $170 \mu \mathrm{g} / \mathrm{ml}$ to fully inhibit protein synthesis. $2 \mathrm{ml}$ of culture was immediately used for analysis in a Varian Cary Eclipse spectrofluorometer (Palo Alto, CA), while the remainder was centrifuged and store at $-80{ }^{\circ} \mathrm{C}$ for immunoblotting. $\mathrm{CFP}$ fluorescence was measured using an excitation wavelength of $434 \mathrm{~nm}$ and an emission wavelength of $477 \mathrm{~nm}$, while YFP fluorescence was measured by using an excitation wavelength of $505 \mathrm{~nm}$ and an emission wavelength of $527 \mathrm{~nm}$. Differences in cell density were corrected for by dividing the fluorescence intensities by the OD $_{600 \mathrm{~nm}}$ of each culture. These values were also corrected for autofluorescnce as observed from MG1655/pEB5 cells.

\section{Single-cell analysis of signal output}

Cells were grown in a similar manner to those used for population-based analysis with slight modifications. As described above, cells were grown overnight at $37^{\circ} \mathrm{C}$ and diluted at least 1:1000 into $3.5 \mathrm{ml}$ of fresh medium with cognate ligand (aspartate or potassium nitrate), control (leucine, isoleucine or potassium chloride) as appropriate. After dilution, cultures were measured directly by flow cytometry (Life Technologies Attune) using a violet (405 nm) and blue (488 nm) laser configuration with detection at $430-470 \mathrm{~nm}$ and 515-545 $\mathrm{nm}$ respectively. A minimum of 50,000 
events for each independent culture were recorded. Compensation was performed by employing strains expressing only CFP or YFP.

Assessing expression of the V5-tagged chimeras

EPB30 cells expressing a chimeric receptor from pRD500 (Taz1), pRD501 (Tez1A1) or pRD502 (NavZ1) were grown as described above to an $\mathrm{OD}_{600 \mathrm{~nm}} \sim 0.3$, harvested by centrifugation and resuspended in $50 \mu \mathrm{l}$ 6X SDS-PAGE sample buffer. Resuspended pellets were subjected to three freeze-thaw cycles of 10 minutes each and then electrophoresed on a 12\% SDS/acrylamide gel. Subsequently, standard conditions were employed for electrophoresis and immunoblotting ${ }^{66}$. During immunoblotting, anti-V5 (Invitrogen) and anti- $\beta$-lactamase (Abcam) were employed as primary antibodies, while Peroxidase-conjugated anti-mouse IgG (Sigma) was used as the secondary antibody. Visulaization and acquisition of the bands was accomplished with ECL Advance Western Blotting Detection Kit (GE Healthcare), a Lumi-Imager F1 Workstation (Roche) and Image Gauge v4.22 software (Fujifilm).

Statistical analysis

All statistical analysis including the one-way ANOVA tests with Tukey-HSD post-hoc analysis were performed using IBM SPSS v23 statistics software (IBM, Armonk, NY, USA). Three asterisks indicate a $p$-value of less than 0.001 .

\section{Supporting information}

Various control experiments described throughout the text have been provided (Figures S1-S3, S5 
and S8). Additional information about the fluorescent output from circuits containing Tar-EnvZ chimeras in the absence (Figures S4 and S6) or presence of aspartate (Figure S7) are also provided. Finally, additional information about the fluorescence output from circuits containing NarX-EnvZ chimeras is shown (Figure S9). This material is available free of charge via the Internet at http://pubs.acs.org.

\section{Acknowledgements}

Members of the Draheim group were very helpful with contributions to the manuscript. Lucy Eke, Robert Lawrence, Thanwarat Chavalwan and Rahmi Yusuf gave the manuscript a thorough proofreading. We thank an anonymous reviewer for critical and insightful comments that helped improve the final output. This work was supported by a grant from the Lundbeck Foundation to M. H. H. N. In addition, start-up funding and the Institute of Biomedical and Biomolecular Sciences (IBBS) at the University of Portsmouth supported R. R. D.

\section{Abbreviations}

MDR, multidrug resistance; TCS, two-component signaling; SHK, sensor histidine kinase; RR, response regulator; VRE, vancomycin-resistant Enterococcus; AIP, autoinducing peptide; GBAP, gelatinase biosynthesis-activating pheromone; CFP, cyan fluorescent protein; YFP, yellow fluorescent protein; TM1, transmembrane helix 1; TM2, transmembrane helix 2; DHp, domain responsible for dimerization and histidylphosphotransfer; CA, domain responsible for catalytic ATPase activity; HAMP, domain originally identified in histidine kianses, adenylate cyclases, MAP kinases and phosphatases; IPTG, isopropyl- $\beta$-D-1-thiogalactopyranoside; SDS-PAGE, sodium dodecyl sulfate-polyacrylamide gel electrophoresis; CW, clockwise; CCW, counterclockwise; MMA, 
minimal medium A; ACDP, advisory committee on dangerous pathogens; PCR, polymerase chain reaction; ECL, enhanced chemiluminescence.

\section{Figure legends}

Figure 1. Harnessing the modularity of TCSs to create chimeric SHKs. (A) Upon stimulus perception by the sensor domain (white) of a canonical SHK, the kinase activity of the CA domain (gray) increases. This results in phosphorylation of the conserved histidyl residue within the DHp domain. Phosphoryl groups are transferred to the aspartyl residue within the received domain of the RR, which usually increases DNA-binding activity of the output domain (black). (B) Canonical SHKs are usually homodimeric and consist of a cytoplasmic (cyto) N-terminus, first transmembrane (TM) helix, a large periplasmic (peri) sensor domain, the second transmembrane helix, the dimerization / histidylphosphotransfer domain (DHp) and the catalytic ATPase (CA) domain. Some SHKs contain additional domains between TM and DHp including HAMP domains. We will exploit conservation within the TM and HAMP domains (depicted in red) to create chimeric SHKs.

Figure 2. Linear and topological composition of the Taz1 and Tez1A1 chimeras. (A) Taz1 is composed of the N-terminus of Tar (white) and the C-terminus of EnvZ (gray) with a fusion joint near the C-terminus of the HAMP domain. Tez1A1 is similar but contains the HAMP domain of EnvZ. Tez1A1 also requires addition of an Ala residue to TM2 for functionality. The purple box indicates the site of aromatic tuning, with the Trp-Tyr tandem responsible for modulation of signal output is highlighted. (B) Topological composition of the Taz1 and Tez1A1 chimeras is the same as in Figure 1B. The location of aromatic tuning and the additional Ala residue required within Tez1A1 are also depicted. 
Figure 3. Response of circuits containing Taz1 or Tez1A1 to aspartate. (A) EPB30 cells expressing Taz1 (circles) or Tez1A1 (triangles) demonstrate an increase in signal output (CFP/YFP) due to increasing aspartate. Leucine (Leu) or isoleucine (Ile) present at $10 \mathrm{mM}$ (gray line) had minimal effect on signal output from either chimera. (B) Fold change from baseline levels of CFP/YFP. Circuits containing Taz1 show an increase in signal output of roughly 30-fold, whereas those expressing Tez1A1 show an increase of only 5-fold, presumably because of higher baseline signal output. Addition of leucine (Leu) or isoleucine (Ile) at $10 \mathrm{mM}$ (gray line) had minimal effect on signal output from either chimera. Error bars represent standard error of the mean with a sample size of $n \geq 3$.

Figure 4. Signal output from circuits containing the aromatically tuned variants of Taz1 and Tez1A1. (A) The primary amino acid sequence where the aromatic tuning was undertaken. The residues contributed by Tar are colored in black, the Trp-Tyr tandem being repositioned is presented in red, and residues contributed by EnvZ are shown in white with a black background. (B) Steady-state signal output of the aromatically tuned Taz1 (circles) or Tez1A1 (triangles) chimeras expressed in EPB30 cells grown under the low- (0\% sucrose, empty) or high-osmolarity (15\% sucrose, filled) regime. Chimeras that exhibited degradation in Figure S5 were not examined. (C) Dynamic range of the various circuits containing the aromatically tuned Taz1 and Tez1A1 chimeras. Error bars represent standard error of the mean with a sample size of $n \geq 3$.

Figure 5. Response to aspartate from circuits containing the aromatically tuned variants of Taz1 or Tez1A1. (A) Changes in signal output (CFP/YFP) from EPB30 cells expressing one of the tuned Taz1 variants (circles, WY-2; triangles, WY-1; squares, wild-type; diamonds, WY+2; pentagons, WY+3) (B) Changes in signal output (CFP/YFP) from EPB30 cells expressing one of the tuned Tez1A1 variants (stars, WY-3; circles, WY-2; triangles, WY-1; squares, wild-type; diamonds, 
WY+2; pentagons, WY+3). (C) Fold change from baseline CFP/YFP in EPB30 cells expressing the wild-type and tuned Taz1 variants. (D) Fold changes from baseline CFP/YFP in EPB30 cells expressing the wild-type and tuned Tez1A1 variants. Error bars represent standard error of the mean with a sample size of $n \geq 3$.

Figure 6. Single-cell analysis of signal output from the tuned Tar-EnvZ chimeras. (A) Response of EPB30/pRD500 (Taz1) cells to no additional ligand (dark gray; MMA), 10 mM aspartate (medium grey; ASP) or $10 \mathrm{mM}$ isoleucine (white; ILE) as analyzed by population-based average in a spectrofluorometer (left panel) or at a single-cell level in a flow cytometer (right panel). (B) Response of EPB30/pRD501 (Tez1A1) cells to no additional ligand (dark gray; MMA), saturating aspartate (medium gray; ASP) or saturating isoleucine (white; ILE) as analyzed by population-based average in a spectrofluorometer (left panel) or at a single-cell level in a flow cytometer (right panel). Error bars represent standard error of the mean with a sample size of $n \geq 3$. To determine statistical significance, one-way ANOVAs with Tukey's HSD post hoc analysis were performed. Three asterisks indicate a $p$-value of less than 0.001 .

Figure 7. Creation and analysis of the NarX-EnvZ chimeras. (A) Linear composition of the NavZ1 chimera. NavZ1 is composed of the N-terminus of NarX (white) and the C-terminus of EnvZ (gray) with a fusion point near the C-terminus of the HAMP domain. NavZ1 requires substitution of a His residue within the HAMP domain to create an $N d e$ I restriction site. The purple box indicates the site of aromatic tuning, with the Trp residue responsible for modulation of signal output underlined. (B) Topological composition of the NavZ1 chimera with the same color scheme and notation as above. (C) CFP and YFP fluorescence from circuits expressing the wild-type or one of the tuned NavZ1 variants. EPB30/pRD502 cells expressing the wild-type NavZ1 exhibited almost no detectable CFP or YFP fluorescence. Dashed lines representing CFP and YFP levels from EPB30/pEB5 (AenvZ) cells 
are provided. (D) The primary sequence where the aromatic tuning is being undertaken. The Trp residue is shown in red and underlined. (E) Steady-state signal output of aromatically tuned NavZ1 chimeras grown under the low- (0\% sucrose, empty circles) or high-osmolarity (15\% sucrose, filled circles) regime. Error bars represent standard error of the mean with a sample size of $n \geq 3$.

Figure 8. Analysis of signal output and ligand-response from tuned NavZ1 chimeras. (A) Steadystate signal output from EPB30/pRD502 (NavZ1) cells grown under the low-osmolarity regime (gray; MMA) and analyzed by spectrofluorometry. (B) Response to no ligand (gray; MMA), $10 \mathrm{mM}$ potassium nitrate (lighter grey; $\mathrm{KNO}_{3}$ ) or $10 \mathrm{mM}$ potassium chloride (white; $\mathrm{KCl}$ ) as analyzed at single-cell level by automated flow cytometry. Error bars represent standard error of the mean with a sample size of $n \geq 3$. To determine statistical significance, one-way ANOVAs with Tukey's HSD post hoc analysis were performed. Three asterisks indicate a $p$-value of less than 0.001 .

\section{References}

1. Blot, S.; Depuydt, P.; Vandewoude, K.; De Bacquer, D., Measuring the impact of multidrug resistance in nosocomial infection. Curr Opin Infect Dis 2007, 20 (4), 391-6.

2. Kirby, T., Europe to boost development of new antimicrobial drugs. Lancet 2012, 379 (9833), 2229-30.

3. Stock, A. M.; Robinson, V. L.; Goudreau, P. N., Two-component signal transduction. Annu Rev Biochem 2000, 69, 183-215.

4. Novick, R. P.; Geisinger, E., Quorum sensing in staphylococci. Annu Rev Genet 2008, 42, 541-64.

5. Cozzone, A. J., An insight into future antibacterial therapy. Emerging microbes \& infections 2012, 1 (11), e38.

6. Mascher, T.; Helmann, J. D.; Unden, G., Stimulus perception in bacterial signal-transducing histidine kinases. Microbiology and molecular biology reviews : MMBR 2006, 70 (4), 910-38.

7. Russo, F. D.; Silhavy, T. J., The essential tension: opposed reactions in bacterial twocomponent regulatory systems. Trends in microbiology 1993, 1 (8), 306-10.

8. Gotoh, Y.; Eguchi, Y.; Watanabe, T.; Okamoto, S.; Doi, A.; Utsumi, R., Two-component signal transduction as potential drug targets in pathogenic bacteria. Curr Opin Microbiol 2010, 13 (2), 232-9.

9. Rasko, D. A.; Moreira, C. G.; Li de, R.; Reading, N. C.; Ritchie, J. M.; Waldor, M. K.; Williams, N.; Taussig, R.; Wei, S.; Roth, M.; Hughes, D. T.; Huntley, J. F.; Fina, M. W.; Falck, J. R.; Sperandio, V., Targeting QseC signaling and virulence for antibiotic development. Science 2008, 321 (5892), 1078-80. 
10. Curtis, M. M.; Russell, R.; Moreira, C. G.; Adebesin, A. M.; Wang, C.; Williams, N. S.; Taussig, R.; Stewart, D.; Zimmern, P.; Lu, B.; Prasad, R. N.; Zhu, C.; Rasko, D. A.; Huntley, J. F.; Falck, J. R.; Sperandio, V., QseC inhibitors as an antivirulence approach for Gram-negative pathogens. mBio 2014, 5 (6), e02165.

11. Rice, L. B., Emergence of vancomycin-resistant enterococci. Emerg Infect Dis 2001, 7 (2), 183-7.

12. Nakayama, J.; Cao, Y.; Horii, T.; Sakuda, S.; Akkermans, A. D.; de Vos, W. M.; Nagasawa, H., Gelatinase biosynthesis-activating pheromone: a peptide lactone that mediates a quorum sensing in Enterococcus faecalis. Mol Microbiol 2001, 41 (1), 145-54.

13. Nakayama, J.; Tanaka, E.; Kariyama, R.; Nagata, K.; Nishiguchi, K.; Mitsuhata, R.; Uemura, Y.; Tanokura, M.; Kumon, H.; Sonomoto, K., Siamycin attenuates fsr quorum sensing mediated by a gelatinase biosynthesis-activating pheromone in Enterococcus faecalis. J Bacteriol 2007, 189 (4), 1358-65.

14. Ma, P.; Nishiguchi, K.; Yuille, H. M.; Davis, L. M.; Nakayama, J.; Phillips-Jones, M. K., Anti-HIV siamycin I directly inhibits autophosphorylation activity of the bacterial FsrC quorum sensor and other ATP-dependent enzyme activities. FEBS Lett 2011, 585 (17), 2660-4.

15. Lyon, G. J.; Mayville, P.; Muir, T. W.; Novick, R. P., Rational design of a global inhibitor of the virulence response in Staphylococcus aureus, based in part on localization of the site of inhibition to the receptor-histidine kinase, AgrC. Proc Natl Acad Sci U S A 2000, 97 (24), 13330-5. 16. Dubrac, S.; Bisicchia, P.; Devine, K. M.; Msadek, T., A matter of life and death: cell wall homeostasis and the WalKR (YycGF) essential signal transduction pathway. Mol Microbiol 2008, 70 (6), 1307-22.

17. Okada, A.; Igarashi, M.; Okajima, T.; Kinoshita, N.; Umekita, M.; Sawa, R.; Inoue, K.; Watanabe, T.; Doi, A.; Martin, A.; Quinn, J.; Nishimura, Y.; Utsumi, R., Walkmycin B targets WalK (YycG), a histidine kinase essential for bacterial cell growth. The Journal of antibiotics 2010, 63 (2), 89-94.

18. Igarashi, M.; Watanabe, T.; Hashida, T.; Umekita, M.; Hatano, M.; Yanagida, Y.; Kino, H.; Kimura, T.; Kinoshita, N.; Inoue, K.; Sawa, R.; Nishimura, Y.; Utsumi, R.; Nomoto, A., Waldiomycin, a novel WalK-histidine kinase inhibitor from Streptomyces sp. MK844-mF10. The Journal of antibiotics 2013, 66 (8), 459-64.

19. Watanabe, M.; Fuda, H.; Jin, S.; Sakurai, T.; Ohkawa, F.; Hui, S. P.; Takeda, S.; Watanabe, T.; Koike, T.; Chiba, H., Isolation and characterization of a phenolic antioxidant from the Pacific oyster (Crassostrea gigas). J. Agric. Food Chem. 2012, 60 (3), 830-5.

20. Beier, D.; Frank, R., Molecular characterization of two-component systems of Helicobacter pylori. J Bacteriol 2000, 182 (8), 2068-76.

21. Zahrt, T. C.; Deretic, V., An essential two-component signal transduction system in Mycobacterium tuberculosis. J Bacteriol 2000, 182 (13), 3832-8.

22. Norholm, M. H.; von Heijne, G.; Draheim, R. R., Forcing the Issue: Aromatic Tuning Facilitates Stimulus-Independent Modulation of a Two-Component Signaling Circuit. ACS Synth Biol 2015, 4, 474-81.

23. Yusuf, R.; Draheim, R. R., Employing aromatic tuning to modulate output from twocomponent signaling circuits. J Biol Eng 2015, 9, 7.

24. Forst, S.; Delgado, J.; Inouye, M., Phosphorylation of OmpR by the osmosensor EnvZ modulates expression of the ompF and ompC genes in Escherichia coli. Proc Natl Acad Sci U S A 1989, 86 (16), 6052-6.

25. Forst, S. A.; Delgado, J.; Inouye, M., DNA-binding properties of the transcription activator $(\mathrm{OmpR})$ for the upstream sequences of ompF in Escherichia coli are altered by envZ mutations and medium osmolarity. J Bacteriol 1989, 171 (6), 2949-55.

26. Lan, C. Y.; Igo, M. M., Differential expression of the OmpF and OmpC porin proteins in Escherichia coli K-12 depends upon the level of active OmpR. J Bacteriol 1998, 180 (1), 171-4. 
27. Russo, F. D.; Silhavy, T. J., EnvZ controls the concentration of phosphorylated OmpR to mediate osmoregulation of the porin genes. J Mol Biol 1991, 222 (3), 567-80.

28. Utsumi, R.; Brissette, R. E.; Rampersaud, A.; Forst, S. A.; Oosawa, K.; Inouye, M., Activation of bacterial porin gene expression by a chimeric signal transducer in response to aspartate. Science 1989, 245 (4923), 1246-9.

29. Zhu, Y.; Inouye, M., Analysis of the role of the EnvZ linker region in signal transduction using a chimeric Tar/EnvZ receptor protein, Tez1. J Biol Chem 2003, 278 (25), 22812-9.

30. Wang, L. C.; Morgan, L. K.; Godakumbura, P.; Kenney, L. J.; Anand, G. S., The inner membrane histidine kinase EnvZ senses osmolality via helix-coil transitions in the cytoplasm. EMBO J 2012, 31 (11), 2648-59.

31. Foo, Y. H.; Gao, Y.; Zhang, H.; Kenney, L. J., Cytoplasmic sensing by the inner membrane histidine kinase EnvZ. Progress in biophysics and molecular biology 2015, 118 (3), 119-129.

32. Ghosh, M.; Wang, L. C.; Ramesh, R.; Morgan, L. K.; Kenney, L. J.; Anand, G. S., LipidMediated Regulation of Embedded Receptor Kinases via Parallel Allosteric Relays. Biophys $J$ 2017, 112 (4), 643-654.

33. Batchelor, E.; Silhavy, T. J.; Goulian, M., Continuous control in bacterial regulatory circuits. J Bacteriol 2004, 186 (22), 7618-25.

34. Heininger, A.; Yusuf, R.; Lawrence, R.; Draheim, R. R., Identification of transmembrane helix 1 (TM1) surfaces important for EnvZ signalling and dimerisation. Biochim. Biophys. Acta 2016, 1858 (8), 1868-1875.

35. Siryaporn, A.; Goulian, M., Cross-talk suppression between the CpxA-CpxR and EnvZOmpR two-component systems in E. coli. Mol Microbiol 2008, 70 (2), 494-506.

36. Michalodimitrakis, K. M.; Sourjik, V.; Serrano, L., Plasticity in amino acid sensing of the chimeric receptor Taz. Mol Microbiol 2005, 58 (1), 257-66.

37. Schultz, J. E.; Natarajan, J., Regulated unfolding: a basic principle of intraprotein signaling in modular proteins. Trends in biochemical sciences 2013, 38 (11), 538-45.

38. Draheim, R. R.; Bormans, A. F.; Lai, R. Z.; Manson, M. D., Tuning a bacterial chemoreceptor with protein-membrane interactions. Biochemistry 2006, 45 (49), 14655-64.

39. Draheim, R. R.; Bormans, A. F.; Lai, R. Z.; Manson, M. D., Tryptophan residues flanking the second transmembrane helix (TM2) set the signaling state of the Tar chemoreceptor.

Biochemistry 2005, 44 (4), 1268-77.

40. Falke, J. J.; Koshland, D. E., Jr., Global flexibility in a sensory receptor: a site-directed cross-linking approach. Science 1987, 237 (4822), 1596-600.

41. Falke, J. J.; Hazelbauer, G. L., Transmembrane signaling in bacterial chemoreceptors. Trends in biochemical sciences 2001, 26 (4), 257-65.

42. Miller, A. S.; Falke, J. J., Side chains at the membrane-water interface modulate the signaling state of a transmembrane receptor. Biochemistry 2004, 43 (7), 1763-70.

43. Falke, J. J.; Erbse, A. H., The piston rises again. Structure 2009, 17 (9), 1149-51.

44. Isaac, B.; Gallagher, G. J.; Balazs, Y. S.; Thompson, L. K., Site-directed rotational resonance solid-state NMR distance measurements probe structure and mechanism in the transmembrane domain of the serine bacterial chemoreceptor. Biochemistry 2002, 41 (9), 3025-36. 45. Botelho, S. C.; Enquist, K.; von Heijne, G.; Draheim, R. R., Differential repositioning of the second transmembrane helices from E. coli Tar and EnvZ upon moving the flanking aromatic residues. Biochim Biophys Acta 2015, 1848 (2), 615-21.

46. Ravid, S.; Matsumura, P.; Eisenbach, M., Restoration of flagellar clockwise rotation in bacterial envelopes by insertion of the chemotaxis protein CheY. Proc Natl Acad Sci U S A 1986, 83 (19), 7157-61.

47. Welch, M.; Oosawa, K.; Aizawa, S.; Eisenbach, M., Phosphorylation-dependent binding of a signal molecule to the flagellar switch of bacteria. Proc Natl Acad Sci U S A 1993, 90 (19), 878791. 
48. Hess, J. F.; Oosawa, K.; Kaplan, N.; Simon, M. I., Phosphorylation of three proteins in the signaling pathway of bacterial chemotaxis. Cell 1988, 53 (1), 79-87.

49. Borkovich, K. A.; Kaplan, N.; Hess, J. F.; Simon, M. I., Transmembrane signal transduction in bacterial chemotaxis involves ligand-dependent activation of phosphate group transfer. Proc Natl Acad Sci U S A 1989, 86 (4), 1208-12.

50. Ward, S. M.; Bormans, A. F.; Manson, M. D., Mutationally altered signal output in the Nart (NarX-Tar) hybrid chemoreceptor. J Bacteriol 2006, 188 (11), 3944-51.

51. Ward, S. M.; Delgado, A.; Gunsalus, R. P.; Manson, M. D., A NarX-Tar chimera mediates repellent chemotaxis to nitrate and nitrite. Mol Microbiol 2002, 44 (3), 709-19.

52. Cheung, J.; Hendrickson, W. A., Structural analysis of ligand stimulation of the histidine kinase NarX. Structure 2009, 17 (2), 190-201.

53. Kitanovic, S.; Ames, P.; Parkinson, J. S., Mutational analysis of the control cable that mediates transmembrane signaling in the Escherichia coli serine chemoreceptor. J Bacteriol 2011, 193 (19), 5062-72.

54. Kitanovic, S.; Ames, P.; Parkinson, J. S., A Trigger Residue for Transmembrane Signaling in the Escherichia coli Serine Chemoreceptor. J Bacteriol 2015, 197 (15), 2568-79.

55. Wright, G. A.; Crowder, R. L.; Draheim, R. R.; Manson, M. D., Mutational analysis of the transmembrane helix 2-HAMP domain connection in the Escherichia coli aspartate chemoreceptor tar. J Bacteriol 2011, 193 (1), 82-90.

56. Bi, S.; Pollard, A. M.; Yang, Y.; Jin, F.; Sourjik, V., Engineering Hybrid Chemotaxis Receptors in Bacteria. ACS Synth Biol 2016, 5 (9), 989-1001.

57. Johnson, B. K.; Abramovitch, R. B., Small Molecules That Sabotage Bacterial Virulence.

Trends in pharmacological sciences 2017, 38 (4), 339-362.

58. Casadaban, M. J.; Cohen, S. N., Analysis of gene control signals by DNA fusion and cloning in Escherichia coli. J Mol Biol 1980, 138 (2), 179-207.

59. Lai, R. Z.; Bormans, A. F.; Draheim, R. R.; Wright, G. A.; Manson, M. D., The region preceding the C-terminal NWETF pentapeptide modulates baseline activity and aspartate inhibition of Escherichia coli Tar. Biochemistry 2008, 47 (50), 13287-95.

60. Adase, C. A.; Draheim, R. R.; Manson, M. D., The residue composition of the aromatic anchor of the second transmembrane helix determines the signaling properties of the aspartate/maltose chemoreceptor Tar of Escherichia coli. Biochemistry 2012, 51 (9), 1925-32.

61. Adase, C. A.; Draheim, R. R.; Rueda, G.; Desai, R.; Manson, M. D., Residues at the cytoplasmic end of transmembrane helix 2 determine the signal output of the TarEc chemoreceptor. Biochemistry 2013, 52 (16), 2729-38.

62. Nour-Eldin, H. H.; Hansen, B. G.; Norholm, M. H.; Jensen, J. K.; Halkier, B. A., Advancing uracil-excision based cloning towards an ideal technique for cloning PCR fragments. Nucleic Acids Res 2006, 34 (18), e122.

63. Norholm, M. H., A mutant Pfu DNA polymerase designed for advanced uracil-excision DNA engineering. BMC Biotechnol 2010, 10, 21.

64. Batchelor, E.; Goulian, M., Robustness and the cycle of phosphorylation and dephosphorylation in a two-component regulatory system. Proc Natl Acad Sci U S A 2003, 100 (2), 691-6.

65. Miller, J. H., A Short Course in Bacterial Genetics: A Laboratory Manual and Handbook for Escherichia coli and Related Bacteria. Cold Spring Harbor Laboratory Press: Plainview, NY, 1992.

66. Ausubel, F. M.; Brent, R.; Kingston, R. E.; Moore, D. D.; Seidman, J. G.; Smith, J. A.; Struhl, K., Current Protocols in Molecular Biology. Wiley: New York, 1998. 
A

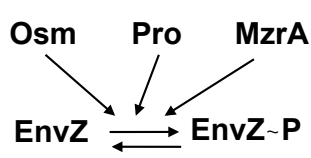

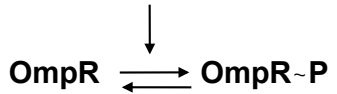

B

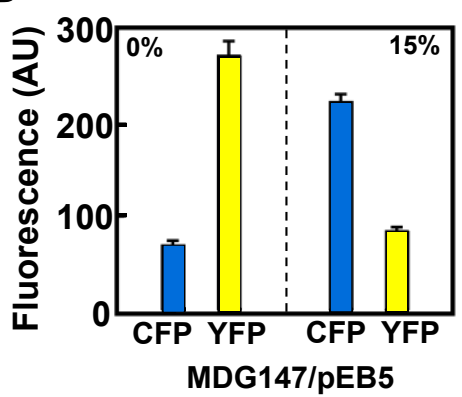

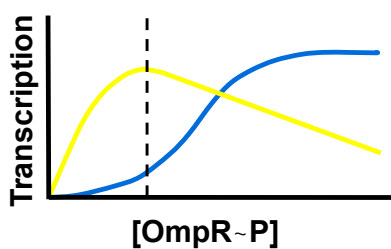

ompF

(YFP) ompC (CFP)

C

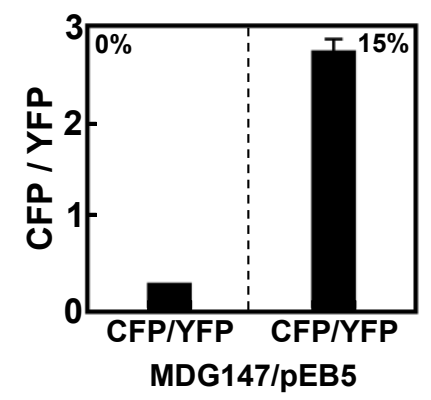

Figure S1. 
A

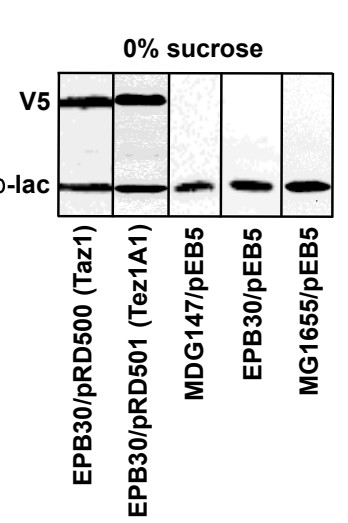

B

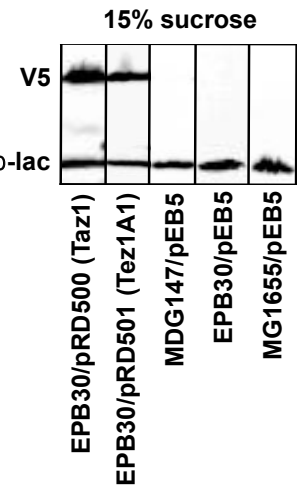

Figure S2. 

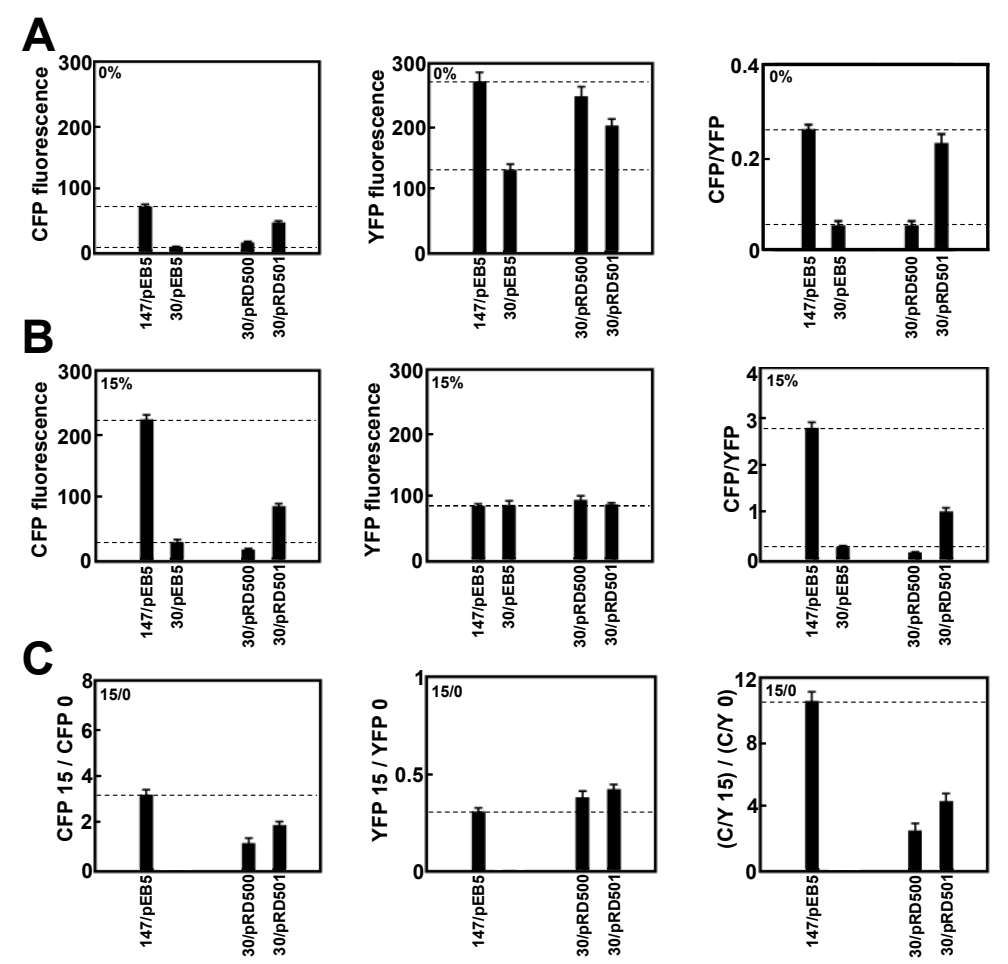

Figure S3. 

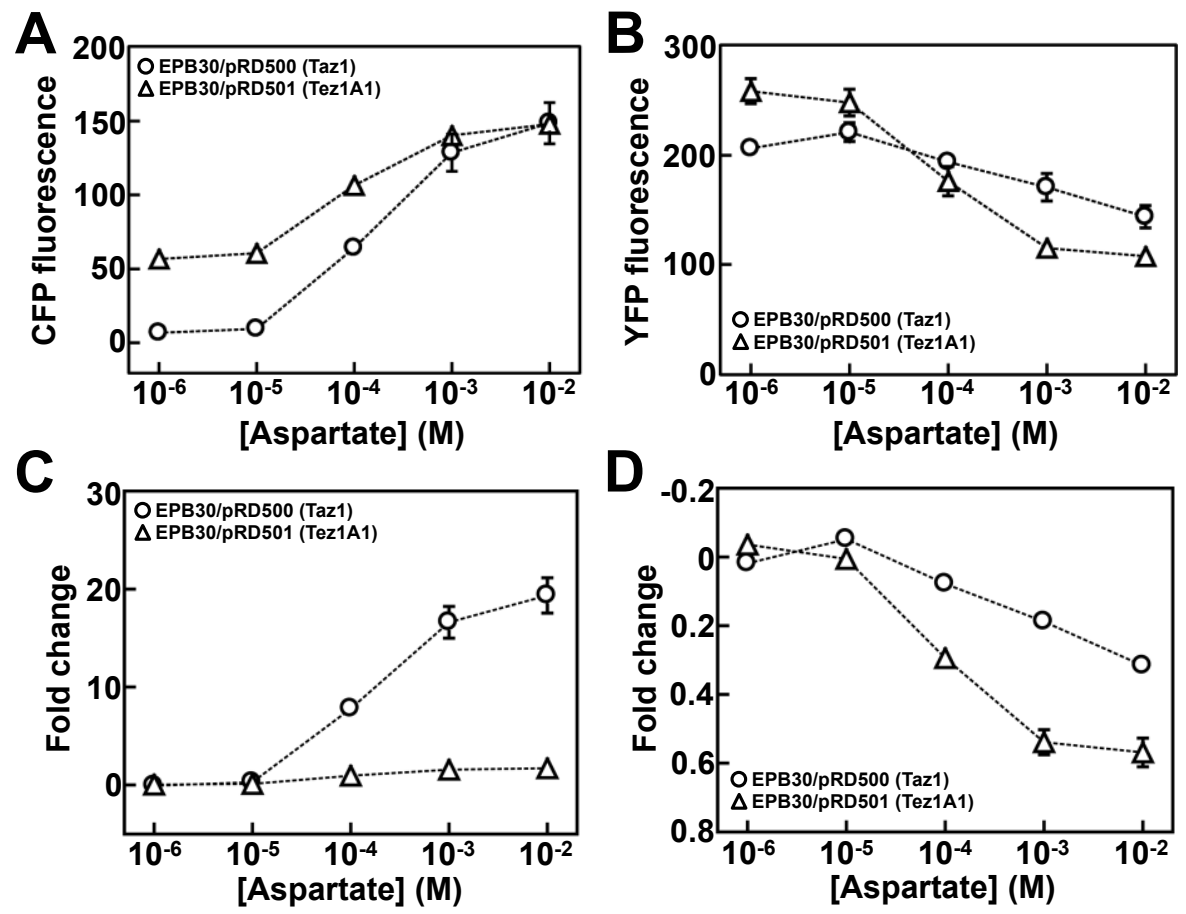

\section{Figure S4.}


A Taz1
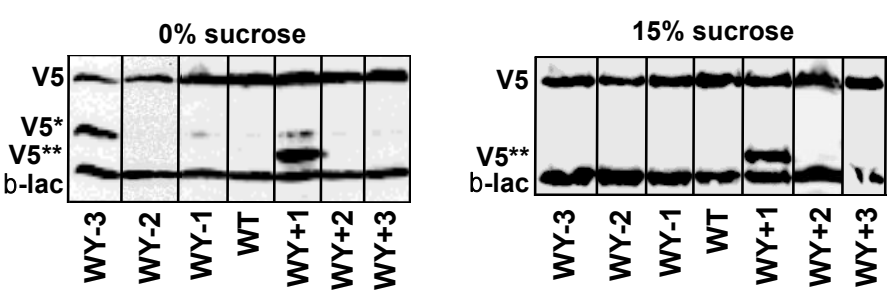

B

Tez1A1
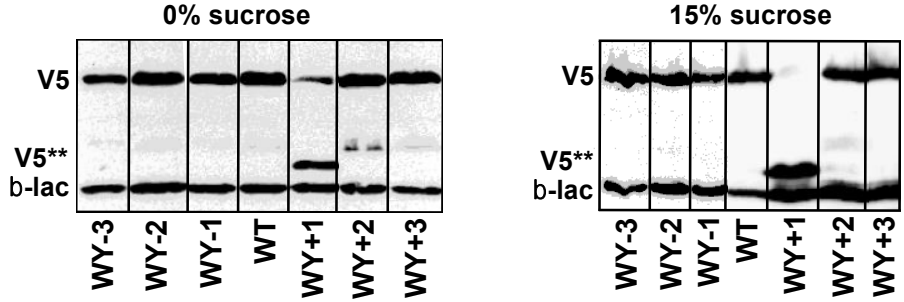

C
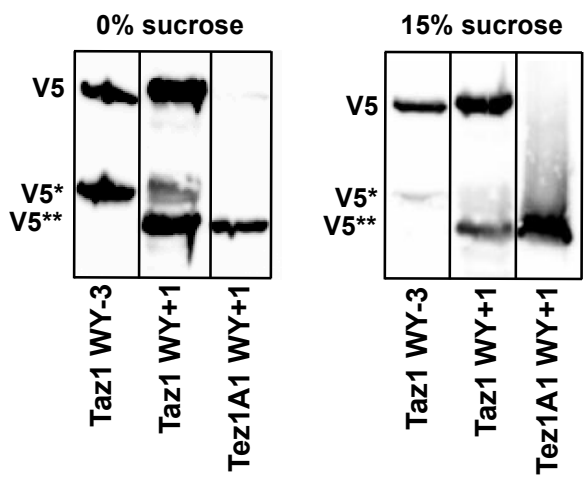

Figure S5. 

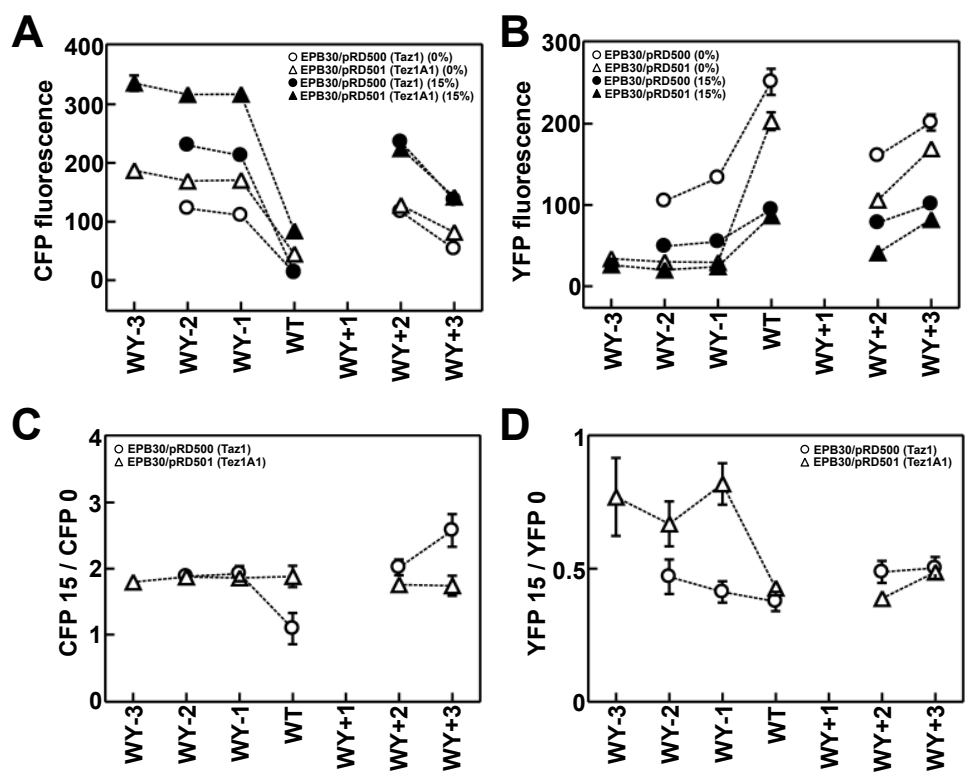

Figure S6. 
A

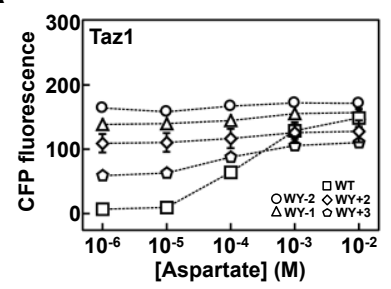

E

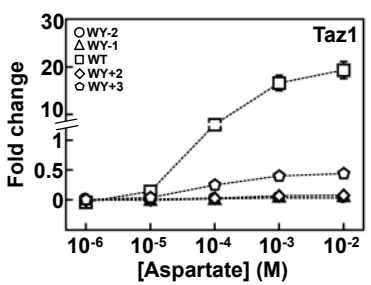

B

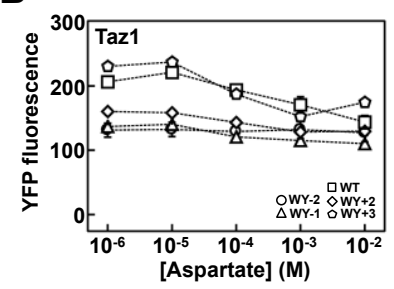

F

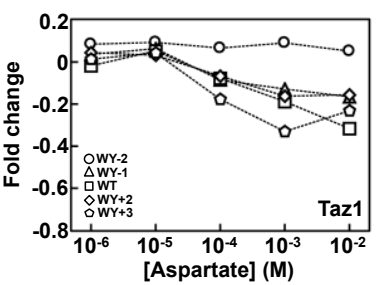

C

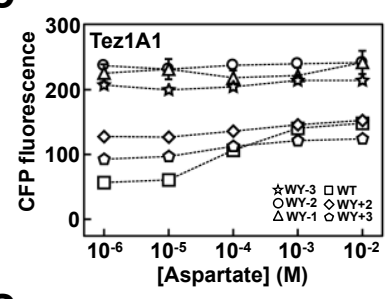

G

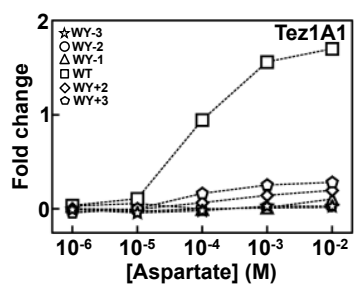

D

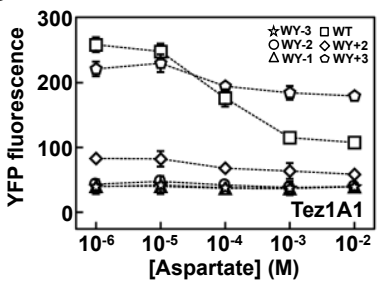

$\mathrm{H}$

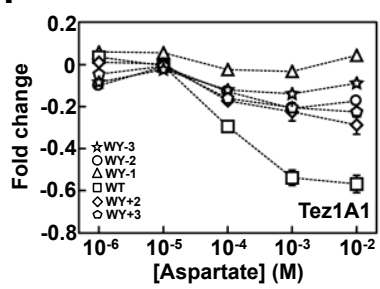

Figure S7. 


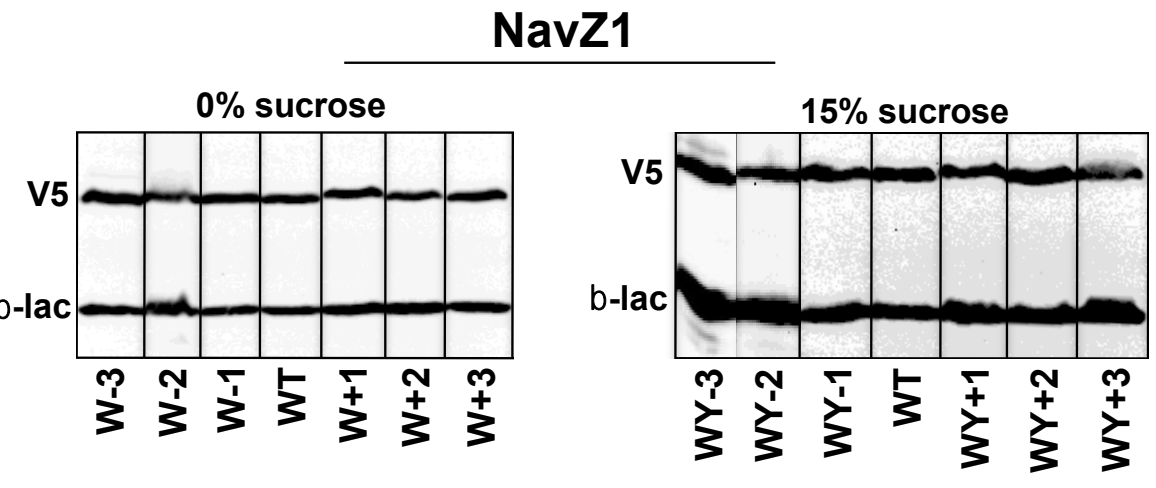

Figure S8. 

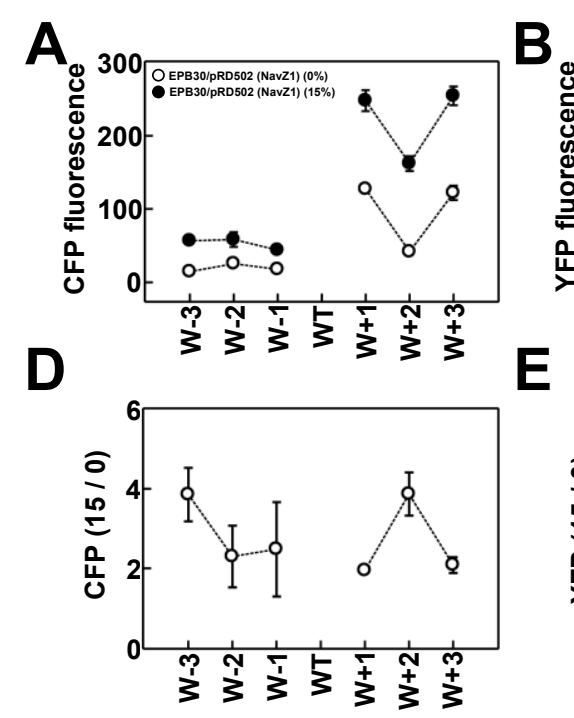

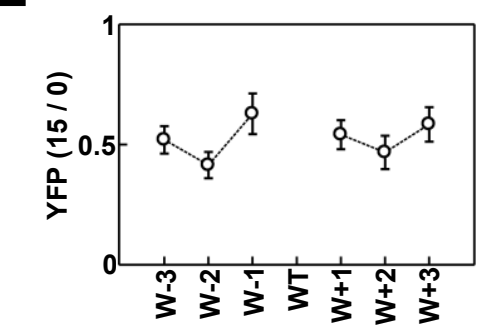

C
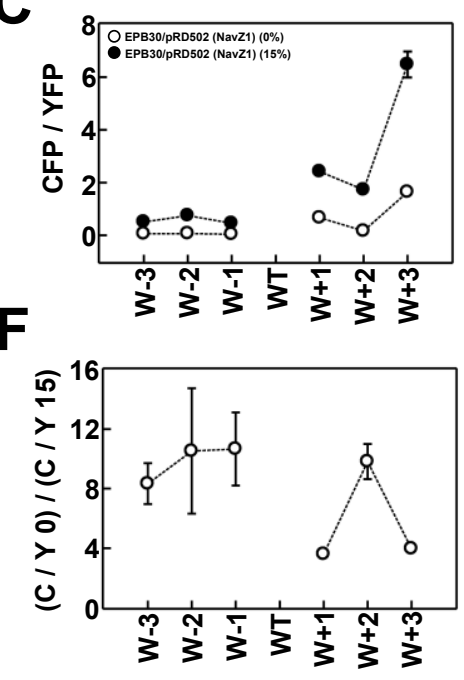

Figure S9. 